\section{Incident reporting in primary care: epidemiology or culture change?}

\section{Susan M Dovey, Katharine A Wallis}

As in most other areas of human experience, fashions in healthcare and in healthcare research come and go. They come about because a good idea is articulated, often as a sound bite that makes intrinsic sense, and they go because a few intrepid researchers are prepared to investigate the substance behind the sound bite and they come to realise that the logic is not supported by the reality. The original logic is supplanted.

Over the last 20-30 years, there have been some notable fashions in healthcare that have lit up the globe like a comet and then died. Just as there have been passing fashions in surgery (eg, routine tonsillectomies) and medications (eg, hormone replacement therapy), so also have there been passing fashions in quality improvement (QI) and QI research. Kieran Walshe in his bibliometric research tracked the frequency paths of different QI terms used between 1988 and 2007.' 'Patient safety' was a relatively low frequency term (along with 'clinical governance' and 'six sigma') from 1988 to 1999 , when suddenly it took off following the release of the Institute of Medicine's 'To Err is Human'2 report, surpassing almost all the other QI terms, along with 'six sigma'.

The rise in evidence-based medicine, arguably itself a fashion, is largely responsible for deposing fash-

Department of General Practice and Rural Health, University of Otago, Dunedin, New Zealand

Correspondence to Dr Susan M Dovey, Department of General Practice and Rural Health, P.O. Box 913, University of Otago, Dunedin, New Zealand; susan.dovey@otago.ac.nz ionable good ideas. Evidence-based medicine, although not immune to criticism, ${ }^{3}$ is different from other fashions in that it has infiltrated every part of every health system - the idea, at least, if not always the practiceand it has not gone away. It is a concept that has captured health systems globally and become embedded as a value, an aspiration and an expectation of healthcare, even more now than it was when the idea first lit up the world more than 40 years ago. Epidemiological research that measures, compares, tests and exposes is the key scientific approach used to create most of the evidence underpinning evidencebased medicine. Epidemiology is so important to medicine now that we are challenged to imagine life before the evidence-based medicine comet.

There may be some justification for viewing the patient safety phenomenon as a paradigm changing one, like evidence-based medicine, but we wonder if the relatively recent focus on incident reporting systems in primary care patient safety research may be a passing (or even passed) fashion. We propose that it is time for patient safety incident reporting to move from being a research tool to being embedded in health systems.

Governments, organisations and institutions have invested huge financial, personnel and time resources in establishing incident reporting systems with a view to identify and remedy threats to patient safety. Processes have been redesigned to make healthcare safer and tested (eg, physician order entry ${ }^{4}$ ), and efforts have been made to understand why known safe practices (eg, handwashing $^{5}$ ) are not always used. However, there is little evidence to show that, overall, health systems are safer places for patients to engage with now than they were 10 years ago. ${ }^{6}$

The early studies identifying particular patient harms, their likelihood of occurrence and degree of preventability were all based in hospitals. ${ }^{7-14}$ These studies have had an impact far in excess of what would seem possible from their size or design. They raised awareness of the harm that healthcare processes can generate and forced critical reviews of health services. They established an epidemiological foundation for threats to patient safety in hospital care, provided an essential foundation for focused patient safety research and prompted health services to investigate and learn from safety management processes in other industries. The aviation industry, in particular, had a history of incident reporting systems that seemed transferable to the health sector and such systems became widely adopted into hospital safety management practices.

Primary care escaped the attentions of patient safety promoters in the early 2000s. The step of establishing an epidemiological description of the patient safety landscape was skipped in primary care. For some, this meant that patient safety was not a primary care issue; for others, it meant that the research just had not been done yet. Taking the latter view, a cohort of primary care researchers, including the authors, set about trying to find out whether people involved in primary care provision should be included when patient safety was being talked about. Emulating the hospital records review studies was considered not only 'cumbersome and costly" ${ }^{15}$ but also impractical. Primary care does not operate in time-limited events like a hospital admission. Primary 
care episodes may unfold over months or years ${ }^{16}$ and primary care patient records may be dispersed across many providers.

However, patient safety reporting systems appeared to be transferable from hospitals into primary care settings. Reporting systems, where providers (in primary care, often doctors) recount the details of the patient safety incidents they observe, have an obvious role to play in contributing to safer healthcare. They provide invaluable insights about the more serious and rare threats to patient safety and give opportunities for clinicians to reflect on care processes they might modify for the good of their patients. They also have disadvantages. While they were initially introduced with a widespread expectation that they could be used to count patient safety incidents and to measure change, in practice they have become more useful as a tool to raise awareness of safety issues and to engage front-line providers in safety management practices. They cannot provide robust epidemiological information that can be used to quantify patient safety risks and measure their health and economic impacts. They cannot monitor patient safety improvements.

Primary care patient safety reporting systems have now been tested in many countries. In primary care, patient safety research has become dominated by research based on reporting systems. ${ }^{17}$ We know now from the extensive repetitions of this research method that they do operate in primary care settings - no further evidence of that is needed. The research reported by O'Bierne $e t$ al is the latest example of primary care reporting systems research, this time from Canada. ${ }^{18}$ However, this research report provides two critical pieces of information that we suspected but did not previously know for sure: first, reporting systems are expensive and, second, people do not like using them.

The reported research was conducted under a finite research grant, so costs could be calculated\$Can 1000 per report. In our view, this is a lot. The actual reports contained little novel information that could not be drawn from existing research and there does not appear to be any reason why family practices in Alberta, Canada, should be broadly different in their experience of patient safety events from any other group of family practices in other parts of the world. However, we appreciate that it is a subjective view.

It is clear from this study that people do not like reporting patient safety incidents to reporting systems and that they therefore provide only a very biased snapshot of all the incidents that actually happen in primary care clinics. Even among practices volunteering to participate in this research and even with the supportive infrastructure that must be in place when research is done, 104 people in the study family practices made only 264 reports in 36 months $(<1$ report per person per year). In a direct observational study, Elder $e t a l^{19}$ reported that in 351 family practice visits where data were collected after each consultation, errors and preventable adverse events were identified in $24 \%$ of encounters. Applying this rate to the Canadian practices suggests that there were, conservatively, around 50000 patient safety events occurring in the Canadian study practices, of which only $0.5 \%$ were reported.

Direct observation is bound to be a more expensive research method than independent report making and therefore impractical to institute on a long-term basis, but we think that primary care patient safety reporting systems have a limited future as a method of research intending to produce epidemiological data. Although they may be a relatively cheap research method to apply, the difficulty in engaging reporters means that they perform poorly for the purpose they are mainly used for-describing primary care patient safety incidents. Moreover, the descriptions that have been reported from them are consistent enough to tell us that the same sorts of problems are encountered in family medicine and general practice clinics wherever reporting systems have been instituted. If they are to be used in future, it should be to address questions relating to their role in behaviour change and making healthcare safer for patients. O'Bierne et $a l^{18}$ suggest that this is where they are heading with their research.

There is now enough evidence that reporting systems, at least primary care reporting systems, are costly and fail to provide the sorts of epidemiological results that really matter. If patient safety incident reporting systems are to become embedded in health systems, following the model of evidence-based medicine, we need to show if and how they make healthcare safer. To date, there has been little research on the role of incident reporting systems as catalysts for culture changes that lead to safer healthcare. This research area needs to be explored further and may be where reporting systems are shown to be most useful. The epidemiology of patient safety incidents in primary care remains a research gap. To explore this, we need to develop other better research methods.

Competing interests None.

Provenance and peer review Commissioned; internally peer reviewed.

Published Online First 19 October 2011

BMJ Qual Saf 2011;20:1001-1003. doi:10.1136/bmjqs-2011-000465

\section{REFERENCES}

1. Walshe K. Pseudoinnovation: the development and spread of healthcare quality improvement methodologies. Int $J$ Qual Health Care 2009;21:153-9.

2. Kohn L, Corrigan J, Donaldson M, eds. To Err Is Human: Building a Safer Health System. Washington DC: National Academy Press, 1999. 
3. Michelson J. Critique of (im)pure reason: evidence-based medicine and common sense. J Eval Clin Pract 2004;10:157-61.

4. Bates D, Leape L, Cullen D, et al. Effect of computerized physician order entry and a team intervention on prevention of serious medication errors. JAMA 1998;280:1311-16

5. Pittet D, Simon A, Hugonnet $S$, et al. Hand hygiene among physicians: performance, beliefs, and perceptions. Ann Intern Med 2004;141:1-8.

6. Landrigan C, Parry G, Bones C, et al. Temporal trends in rates of patient harm resulting from medical care. $N$ Engl $J$ Med 2010;363:2124-34.

7. Brennan T, Leape L, Laird N, et al. Incidence of adverse events and negligence in hospitalized patients. Results of the Harvard Medical Practice Study I. N Engl J Med 1991;324:370-6.

8. Wilson R, Runciman W, Gibberd R, et al. The Quality in Australian Health Care Study. Med J Aust 1995;163:458-71.
9. Thomas E, Studdert D, Burstin $\mathrm{H}$, et al. Incidence and types of adverse events and negligent care in Utah and Colorado. Med Care 2000;38:261-71.

10. Vincent C, Neale G, Woloshynowych M. Adverse events in British hospitals: preliminary retrospective record review. BMJ 2001;322:517-19.

11. Baker G, Norton P, Flintoft V, et al. The Canadian Adverse Events Study: the incidence of adverse events among hospital patients in Canada. CMAJ 2004;170:1678-86.

12. Davis $\mathrm{P}$, Lay-Yee $\mathrm{R}$, Briant $\mathrm{R}$, et al. Adverse events in New Zealand public hospitals II: preventability and clinical context. $N Z$ Med $J$ 2003;116:U624.

13. Davis $\mathrm{P}$, Lay-Yee $\mathrm{R}$, Briant $\mathrm{R}$, et al. Adverse events in New Zealand public hospitals I: occurrence and impact. $N Z$ Med J 2002;115:U271.

14. Schiøler T, Lipczak H, Pedersen B, et al; Danish Adverse Event Study. [Incidence of adverse events in hospitals. A retrospective study of medical records] (In Danish) Ugeskr Laeger 2001;163:5370-8.

15. Neale G, Woloshynowych M. Retrospective case record review: a blunt instrument that needs sharpening. Qual Saf Health Care 2003;12:2-3.

16. Lamberts $\mathrm{H}$, Hofmans-Okkes I. Episode of care: a core concept in family practice. $J$ Fam Pract 1996;42:161-7.

17. Makeham M, Dovey S, Runciman W, et al. Methods and Measures used in Primary Care Patient Safety Research: Results of a literature review. 2009. http://www.who. int/patientsafety/research/methods measures/makeham_dovey_full.pdf

18. O'Beirne M, Sterling $\mathrm{P}$, Zwicker $\mathrm{K}$, et al Safety incidents in family medicine $B M J$ Qual Saf 2011;20:1005-10.

19. Elder N, VonderMeulen M, Cassedy A. The identification of medical errors by family physicians during outpatient visits. Ann Fam Med 2004;2:125-9. 